\title{
An Efficient and Reflective Event Filtering for Context-Awareness in Ubiquitous Computing
}

\author{
Kyu Bong Cho ${ }^{1}$, Sung Keun Song ${ }^{1}$, Hee Yong Youn ${ }^{1}$, and Gyung Leen Park ${ }^{2}$ \\ ${ }^{1}$ School of Information and Communications Engineering \\ Sungkyunkwan University, Suwon, Korea \\ mongbong@skku.edu, skk103@devg.org, youn@ece.skku.ac.kr \\ ${ }^{2}$ Department of Computer Science and Statistics \\ Cheju National University, Cheju, Korea \\ gpark@cheju.ac.kr
}

\begin{abstract}
In the ubiquitous computing system fast and reliable event delivery is important to properly adapt to the dynamically changing environment. Such requirement could be satisfied by message-based middleware based on the publish/subscribe model. In this paper we propose a scheme that allows reflective filtering through the reasoning process. The objective of the reflective filtering is to prevent waste in the space of the event queue of the event channel due to useless events and reduce the network load. Performance evaluation reveals that the proposed scheme displays a significant improvement over the existing event service, Java messaging service (JMS) and omnievent service, in terms of event delivery time.
\end{abstract}

Keywords: Context-oriented filtering, event service, message-oriented middleware, publish/subscribe, reflective filtering.

\section{Introduction}

The ubiquitous computing for any-time, any-where, any-network, any-device, and any-service is becoming a core paradigm of the modern information-oriented society. In such environment the post PC-type computers provide various services useful to human by using real-time context information of the objects [1]. Here reliable and fast communication must be guaranteed to adapt to the dynamically changing environment and receive the context data of the environment including the event data $[2,3]$.

In the ubiquitous environment the basic mechanisms employed for event transmission are remote procedure call (RPC), remote method invocation (RMI), and message- oriented middleware (MOM), etc. The RPC offers an abstraction of high level, but it does not guarantee reliability in addition to static binding and limited scalability. To solve the problem while dealing with the heterogeneous environment of various programming languages, hardware platforms, and operating systems, the MOM based on message queuing was developed. Later, the distributed object computing paradigm providing more abstraction and transparency of users appeared. There also exist peer-to-peer, publish/subscribe, and hierarchical channel model as the messaging scheme for event transmission. The common characteristics of various MOM 
products and the publish/subscribe model based on message queuing and filtering are presented in $[4,5]$.

The publish/subscribe model among various messaging models is a representative event transmission model used widely. The current popular event-based transmission services are JMS (Java Message Service) [7], CORBA event service [9], and CORBA Notification service [6]. They employ filtering to filter unwanted events out. However, the overhead of filtering in the Notification service has been recognized to be very high because filtering occurs in several proxies. Note that the JMS needs JVM, which is an additional overhead of Java-based system with respect to the speed of event delivery. The JMS handles message filtering in the server like CORBA Notification service, and thus the overhead in the processing and managing the messages in the queue is high [8].

In this paper we propose a reflective filtering approach where the filtering criteria of the client are sent to the supplier through a reasoning process. The approach can enhance the capability of event service using the algorithm of highly efficient and scalable reflective message filtering [9]. It implements context-awareness for handling the requests generated from the environment the users belong to. The objective of the proposed reflective filtering is to prevent the waste in the space of the event queue of the event channel due to useless events and reduce the network load. Extensive experimentation reveals that the proposed approach allows consistently higher performance than the JMS and CORBA event service in terms of event delivery time for various numbers of servers and data sizes. Also, it reveals that the performance significantly varies according to where the filtering is applied.

The rest of the paper is organized as follows. Section 2 presents the related work and Section 3 introduces the proposed event filtering approach. The proposed approach is evaluated in Section 4, and conclusion is made in Section 5.

\section{The Related Work}

\subsection{Classification of Filtering Algorithms}

Several algorithms for distributed filtering have been proposed. Evaluation and comparison of the algorithms are difficult due to the diversity of the approaches. What is needed is a concise classification of the approaches employed for distributed filtering [10].

Location of Filtering: Filtering can be performed at the subscribers (flooding of events) or providers (flooding of profiles), or at certain broker nodes. Flooding of events results in high network traffic, but requires small memory space. Flooding of profiles, however, results in the opposite: less network traffic but large memory space. Filtering at fixed brokers allows the advantage of filtering control according to available resources, but has the disadvantage of high load at the filtering brokers in terms of network traffic and memory usage.

Spreading of Filtering Complexity: The filtering complexity can be spread over several brokers by exclusive filtering at certain brokers or by distributed filtering. Exclusive filtering can be implemented with some control overhead. A disadvantage 
of exclusive filtering is the danger of multiple notifications for a single event because the event notification may be forwarded to several neighbor brokers.

Communication with the Subscribers: In direct communication only the filtering broker and the subscriber are involved in the communication. A disadvantage here is that either a connectionless protocol has to be used (resulting in unreliable communications) or new connections have to be established over the time. When forwarding messages via the network of brokers, only the neighbor brokers and local clients communicate directly. Local clients are the publishers and subscribers that are directly connected to a broker. A drawback here is high memory usage. When using brokers as the proxies, the brokers act as the subscribers to their neighbor nodes and thus the number of subscribers each broker node has to deal with in limited. Exploiting the coverage between the profiles of several subscribers is possible and beneficial in several aspects. A disadvantage, though, is the necessity of post-filtering in the notification to the client subscribers.

\subsection{Java Message Service (JMS)}

The Java Message Service is a Java API that allows the applications to create, send, receive, and read the messages. A JMS provider is a messaging system that implements the JMS interfaces and provides administrative and controlling features. The two kinds of administered objects are destinations and connection factories. The technology underlying these objects is likely to be very different from one implementation of the JMS API to another. Therefore, management of the objects belonging to different administrative tasks varies from provider to provider. The JMS clients access the objects through portable interfaces, and therefore a client application can be run with little or no change on more than one implementation of the JMS API. Figure 1 illustrates the way the components in the JMS interact with each other.

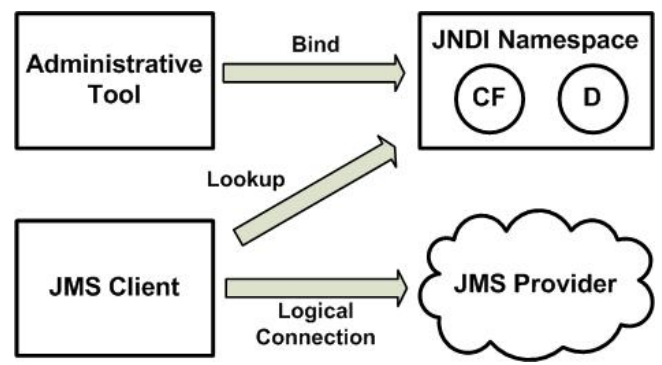

Fig. 1. The JMS API architecture

The JMS message is composed by a header, properties, and body, and it is typed based on the data contained in the message body. The class hierarchy of the JMS message is organized such that the Message interface, which is the root interface for all the JMS message types, defines the header fields, property facility, and associated accessor methods. A JMS message selector is used by a JMS client to specify which messages it is interested in. It is based on the values contained in the message header, which includes standard header fields as well as optional fields that are added via 
application, standard, or provider-specific properties. As noted earlier, a message selector cannot refer to the value of the message body. A message selector is a Boolean expression that, when it evaluates to be true, results in the matched message to be passed to the client. It is defined as a string, and its syntax is based on a subset of the SQL92 conditional syntax. Creating a selector involves the generation of a string that conforms to the defined syntax [7].

\section{The Proposed Event Filtering}

The proposed event filtering is a part of a new reflective context-oriented event service embedded in the CALM (Component-based Autonomic Layered Middleware) research project [11]. We first briefly introduce the CALM.

\subsection{CALM}

The CALM adopts the reflective paradigm and agent system to construct a flexible and efficient middleware platform for ubiquitous computing. It also provides useful development tools. It consists of two internal layers, one external layer, and various tools forming an efficient agent-based platform. The internal layer consists of the communication platform layer and agent platform layer. The communication platform layer is constructed such that it can provide various distributed services based on the context and situation using appropriate communication protocols and devices in wired and wireless environment. The agent platform layer is composed using the components so that it can maximize the efficiency of the service, adapt itself to the environment, and accommodate the advantage of diverse agent systems. The external layer is composed by self-growing engine and ontology-based situation-awareness engine required for providing intelligent services.

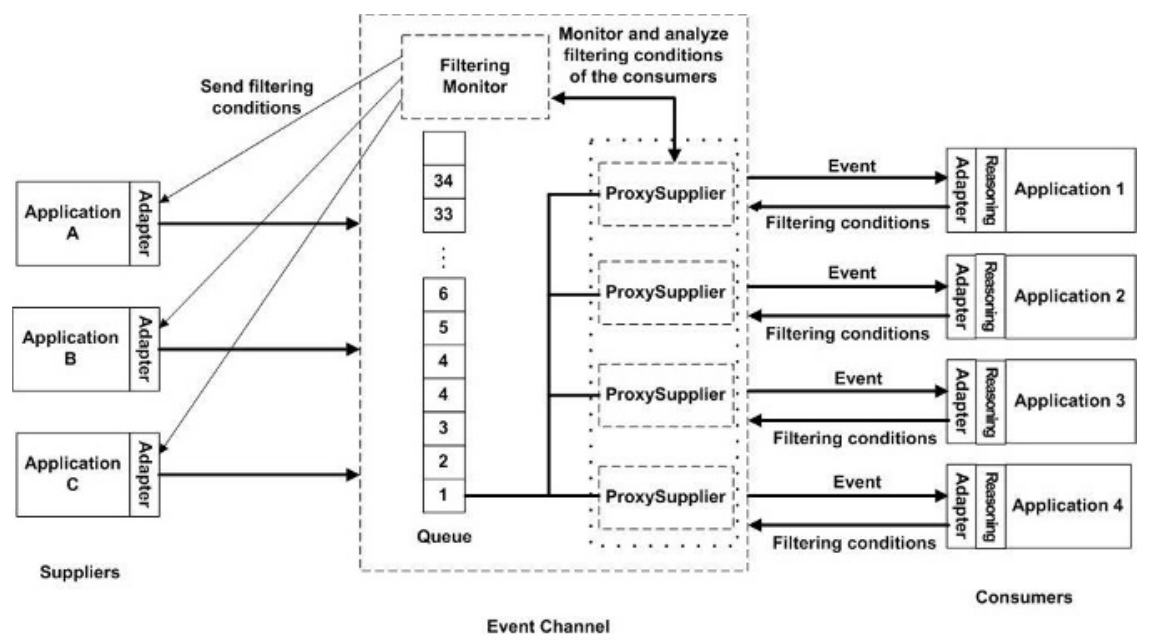

Fig. 2. The structure of the proposed reflective filtering 


\subsection{Reflective Filtering}

The objective of reflective filtering is to prevent waste in the space of the event queue of the event channel due to useless events and reduce the network load. The concept of reflective filtering process is shown in Figure 2. The consumers decide the filtering criteria with which each consumer declares what kinds of context information are desired to be received through reasoning. The adapter of the consumers connects it to the event channel and sends its own filtering criteria to relevant proxysuppliers. The filtering monitor of the event channel monitors and analyzes the filtering criteria of the consumers and sends the result of analysis to the adapters of the suppliers. The filtering monitor is normally in sleeping mode and activates itself upon receiving a new filtering criterion from a consumer or a new consumer connects to the event channel. The adapter of the suppliers receives the filtering criteria from the filtering monitor of the event channel. The adapter of the suppliers filters out the events that the application has not indicated to receive from the event channel.

\subsection{Reliable Communication}

The proposed event service employs event numbering and event queues to support reliable communication. The format of an event of the proposed event service is as follows;

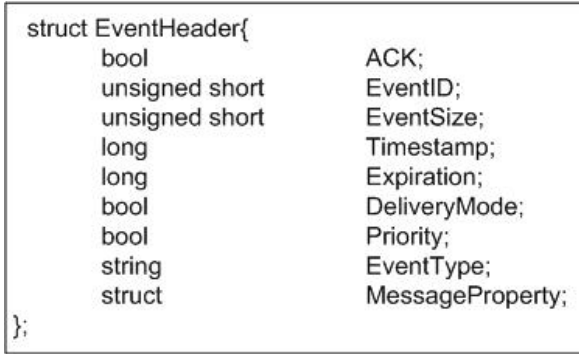

The event channel and adapter have an event queue, respectively. Reliable communi-cation is provided as follows; the adapter of the supplier stores the events at its own event queue before sending them to the event channel. Thereafter, it sends the events to the event channel. It waits for an acknowledgment from the event channel after sending the events. If the adapter receives an ACK from the event channel and the event queue is full, it overwrites the Acked events with new events until the events are Acked. In case that the adapter receives a retransmission request from the event channel, it resends the events stored in the event queue.

Efficient message filtering needs an effective design of header and payload of the message. Message is the most important part in message filtering and the message format is shown in Figure 3. The events communicate with each other using the messages carrying the data of applications, and different components of the adapter are used in message transmission. The components compose an alive system. 


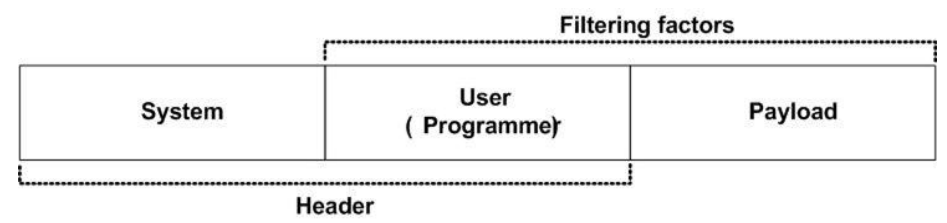

Fig. 3. The message format

A message object is divided into two parts, message header and message data itself (payload). The messages have several different types decided based on the payload of the message. The payload is StreamMessage, MapMessage object, or takes a standardized TextMessage, ObjectMessage and BytesMessage type. A message carries data, and it is also used as a simple means of notification of the events occur-ing in the system.

The message header supplies the MetaData describing who created the message, when it was created, and how long it is valid. Also, a message can include the attribute that can be defined and set by the client. The subscribers can filter the message through this attribute and select the messages according to the value of the specification header and payload. The message attribute is put in the additional header that can be attached to the message, and it allows the application developer to include more information in a message.

\section{Performance Evaluation}

The performance of the proposed approach is carried out concerning message delivery time, and it is compared with the omnievent service and JMS. The test platform includes twelve identical Windows XP-based PCs, each with one 2.1-Ghz CPU and

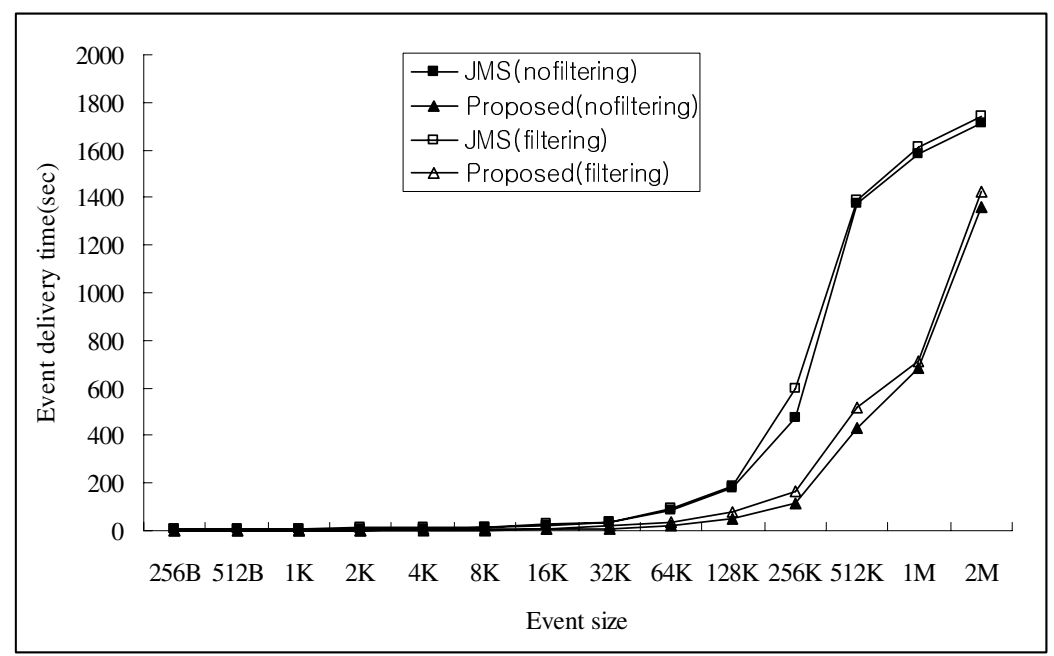

Fig. 4. The comparisons of event delivery time for one-to-one connection 
512-Mbyte RAM. In case of the proposed reflective filtering and omnievent service ten PCs host suppliers, one PC hosts the naming server, and one PC hosts the consumer. In case of the JMS ten PCs are senders (suppliers), which connect to their local server and a PC is their subscriber (consumer). Figure 5 and 6 show the average event delivery time of 1000 test runs in case of one-to-one and many-to-one connection, respectively.

In Figure 4 each supplier generates 2000 context information while the event size is increased up to $2 \mathrm{Mb}$. Notice from the figure that filtering allows faster delivery than the case of nonfiltering for both the systems. Also, notice that the proposed event service displays always smaller delivery time while the improvement gets more significant as the event size grows. Figure 5 shows the filtering efficiency when the number of suppliers is increased. Again, the proposed scheme shows a significant improvement because filtering occurs in the JMS server while the suppliers transmit data without considering the consumer's preference. Note that the proposed event service applies filtering in the supplier. We obtain the same result with the omnievent service, which is not included here due to space limit.

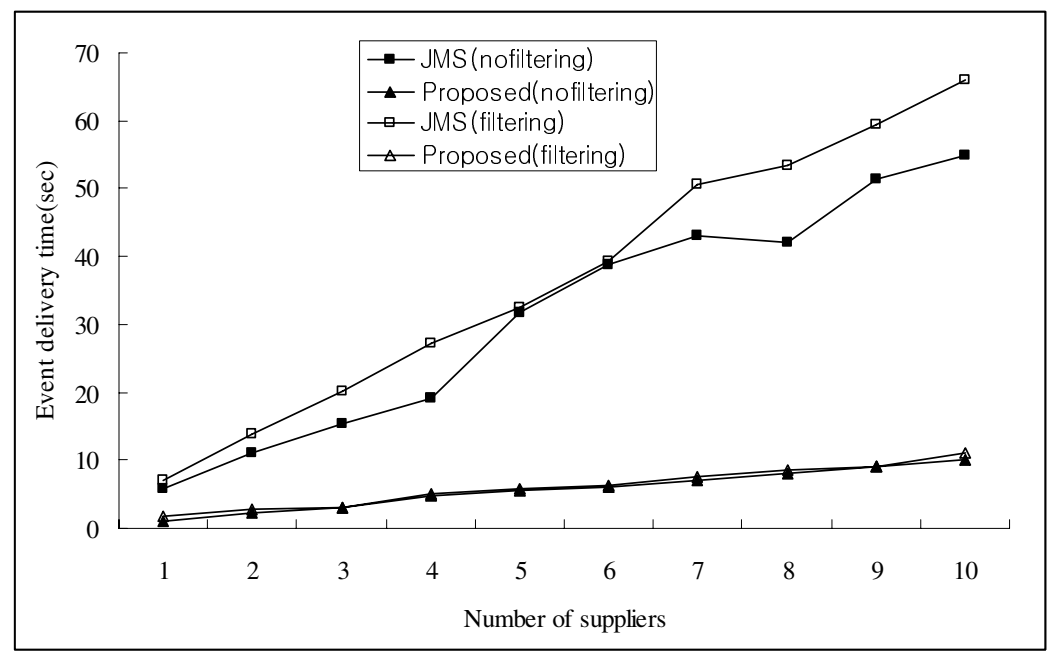

Fig. 5. The comparisons of event delivery time for many-to-one connection

\section{Conclusion and Future Work}

In the pervasive computing the importance of event filtering is very high since a large number of events are generated in the environment. Various distributed filtering algorithms have been proposed for the publish/subscribe based systems, but systematic analysis and comparison of them is difficult. In this paper we have proposed a context-oriented event filtering approach supporting reliable communica-tion and reflective filtering through the adapters. The proposed event filtering is implemented in the CALM developed by the authors. It also reduces the network load of CORBAbased event servers which is an important requirement for large scale ubiquitous 
environment. Extensive computer simulation reveals that the proposed approach allows consistently higher performance than other approaches in terms of event delivery time for various number of servers and data sizes. We will further investigate the filtering approaches dynamically adaptable for various operational conditions and requirements.

\section{References}

1. Ha, W. Ubiquitous IT revolution and the third space Korean Elictrinic, 2002.

2. Karen, H. and Jadwiga, I. A Software Engineering Framework for Context-Aware Pervasive computing. Proceedings of the Second IEEE Annual Conference on Pervasive Computing and Communications (PERCOM'04), pp. 77.

3. Schantz, R. E. and Schmidt, D. C. (2001) Middle-ware for Distributed Systems: Evolving the Common Structure for Network-centric Application Encyclopedia of Software Engineering, New York, Wiley \& Sons pp. 801-813.

4. Astley, M., Sturman, D. and Agha, G. Customizable middleware for modular distributed software. Commun ACM 44, 5 (May 2001), pp. 99-107.

5. Coulson, G. What is reflective middleware? IEEE Distribute System Online 2, 8 (Dec. 2001); see computer.org/dsonline/middleware/RMarticle1.htm.

6. Object Mgmt. Group Notification Service Specification OMG Doc. Telecom/99-07-01 ed., July 1999.

7. http://java.sun.com/products/jms/tutorial/

8. Lan, G. et al. An Efficient, Scalable Content-Based Messaging System. Proceedings of the seventh IEEE International Enterprise Distributed Object Computing conference (EDOC'03), pp. 278-285.

9. Object Management Group Event Service Specifi cation Version 1.2, October 2004.

10. Sven, B. and Annnika, H. Classification and Analysis of Distributed Event Filtering Algorithms. Proceedings of Cooperative Information Systems (CoopIS) 2004 International Conference, pp. 301-318.

11. Youn, H.Y. et Al. CALM: An Intelligent Agent-based Middleware Architecture for Community Computing, Proceedings of the third Workshop on Software Technologies for Future Embedded \& Ubiquitous Systems (SEUS 2006). 